\title{
Cutaneous cryptococcosis: an underlying immunosuppression? Clinical manifestations, pathogenesis, diagnostic examinations and treatment
}

\author{
Marcela A. Nowak ${ }^{1}$, Anna Putynkowska², Wioletta Barańska-Rybak ${ }^{1}$, Kamila Czarnacka ${ }^{1}$, Maria A. Dębska-Ślizień ${ }^{1}$ \\ ${ }^{1}$ Department of Dermatology, Venereology and Allergology, Medical University of Gdansk, Gdansk, Poland \\ 2Department of Dermatology, Copernicus Hospital, Gdansk, Poland \\ Adv Dermatol Allergol 2020; XXXVII (2): 154-158 \\ DOI: https://doi.org/10.5114/ada.2020.94833
}

\begin{abstract}
Due to constantly growing population of immunocompromised patients the fungi became a widespread threat to modern medicine. HIV carriers, solid organ transplant recipients constitute most of those patients. Cryptococcosis is a frequent cause of life-threatening infections, affecting mostly immunosuppressed patients. This article presents current knowledge on cryptococcal infections, including epidemiology, clinical aspects, diagnosis and recommended treatment. In reference to our patient, who developed a disseminated and fulminant subtype of the disease, we wanted to underline the need to examine patients thoroughly. The highest aim of those measures would be to avoid lethal consequences.
\end{abstract}

Key words: cryptococcosis, Cryptococcus neoformans, kidney transplantation, dermatomycoses.

\section{Introduction and epidemiology}

Cryptococcosis is a systemic, fungal, life-threatening infection caused by encapsulated yeast-like fungus Cryptococcus neoformans (C. neoformans), a compelling pathogen which can be found worldwide [1]. Humans can get infected by inhaling pigeon excreta, dropping, soil or even fruits, which may harbour the yeast. Already in 1895 Busse and Buschke described the same case of a woman with an ulcer on her lower limb. During the histopathological examination of this skin lesion Cryptococci forms were found and described in the literature for the first time [2].

A rise in cryptococcosis cases has been reported since the 1950s, due to constantly growing population of immunocompromised patients. Cryptococcosis mostly applies to those patients, in particular HIV-positive and solid organ transplant recipients [3]. Primarily the respiratory tract, central nervous system, skin, prostate and bones are affected.

Common pathogens responsible for cryptococcosis include Cryptococcus neoformans and Cryptococcus gatti. Those fungi are currently the leading cause of fungal meningitis worldwide [4]. C. neoformans primarily infects patients with a low CD4+ T cell count, whereas C. gatti has the ability to infect also immunocompetent individuals $[5,6]$. As inhalation of spores initiates cryptococcal infection, the first organs to be affected are usually lungs [7]. In healthy individuals, whose immune system is functioning in an undisturbed way, the disease might limit only to asymptomatic respiratory infection. However, in immunocompromised individuals the infection may result in severe pneumonitis and spread to the central nervous system and other locations such as the skin, prostate or bones. This is owing to fungistatic and fungicidal host defence mechanisms, which include activation of the humoral system through the complement cascade [8]. Furthermore, the disease is more frequent among men, which suggests oestrogen's protective effects [9].

\section{Clinical manifestations}

Most commonly cryptococcosis is found primarily in lungs. Chest $X$-rays reveal unilateral, nodular or cavitary infiltrates [10]. Pleural effusion is relatively uncommon. Depending on the immune status of the individual, pulmo-

Address for correspondence: Marcela A. Nowak, Department of Dermatology, Venereology and Allergology, Medical University of Gdansk, 3 Marii Skłodowskiej-Curie St, 80-001 Gdansk, Poland, e-mail: mcela94@gmail.com Received: 6.08.2018, accepted: 2.09.2018. 
nary cryptococcosis ranges from asymptomatic infection to severe pneumonia and acute respiratory failure [11].

From lungs, the fungus disseminates primarily to the central nervous system. The symptoms are highly unspecific and include headache, memory loss, tremor, muscle weakness, disorientation and confusion. Preferentially basal meninges are affected, which leads to their thickening with consecutive spread to deeper brain tissues [12]. In the meninges themselves, Cryptococcus is suspended in a capsule-derived mucous membrane. The dissemination is possible owing to serious defects in cell-mediated immune defence. Among risk factors advanced AIDS, corticosteroids use, lymphomas, solid organ transplants are the most essential. Literature gives also familial cryptococcosis cases, when the prevalence of the disease is due to genetic defects in the immune system [13].

Cutaneous cryptococcosis, due to haematogenous spread, concerns around $20 \%$ of patients with disseminated cryptococcosis. Moreover C. neoformans serotype D is associated with an even higher risk of developing the cutaneous form, due to its dermatotropism [14].

Cryptococcosis can cause almost any type of the cutaneous lesion from papules, acneiform lesions, ulceration to nodules [15]. A typical lesion appears as a dome-shaped papule with umbilicated centre [16]. It can also mimic other dermatological entities, such as bacterial cellulitis, sarcoidosis or panniculitis. In patients with AIDS lesions usually resemble molluscum contagiosum. Therefore a thorough examination of such lesions should always be carried out as they may be the first clinical signs of cryptococcal infection and its dissemination [17]. The frequency of skin involvement in cryptococcosis is significantly higher in liver transplant recipients receiving tacrolimus [4].

In May 2015, a 65-year-old patient with disseminated cryptococcosis was referred to the Clinic of Nephrology

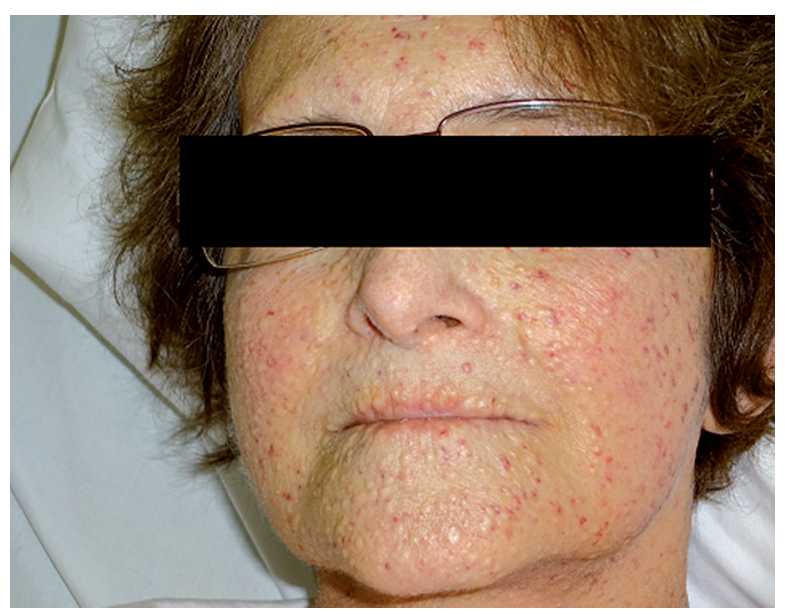

Figure 1. Skin changes (papules with umbilicated centre) in our patient with cryptococcosis and Transplantology at the University Clinical Centre in Gdansk, Poland. The patient presented unique cutaneous lesions. She was receiving immunosuppressive treatment, due to recent renal transplantation. Dermatological consultation revealed erythematous, solid, regular and well-defined papules on the skin (affected by telangiectasia) of the whole face (Figure 1) with some petechiae among them. Biopsy specimens were obtained from one of the papules on the face. A microscopic examination demonstrated fine-grained granulomatous concentrations surrounded by fragments of elastoid stroma. Staining with Alcian blue and mucicarmine affirmed presence of cryptococcal organisms (Figure 2).

Based on clinical, microbiological and histopathological findings, the disseminated form of cryptococcosis with secondary cutaneous involvement was diagnosed. Assuming that renal graft was the primary source of fungemia, an urgent graftectomy was carried out. Antifungal treatment, which consisted of $150 \mathrm{mg}$ of intravenous liposomal amphotericin B together with 2500 mg of flucytosine was implemented.

Histopathological examination of the removed graft demonstrated many fungal colonies after Grocott staining, mostly in macrophages, reaffirming cryptococcosis diagnosis.

After graftectomy our patient was haemodialysed every day. However, despite intravenous antifungal treatment and antibiotic therapy, the patient's general condition was constantly worsening. At that time, the patient presented hectic fever and progressive cachexia. C. neoformans was present in the blood cultures only for a short time after graftectomy, yet the serum antigen level was increasing.

We asked for PET examination as there was no certain source of this prolonged, drug-resistant infection. To our surprise, PET result revealed an active metabolic process in the thyroid (suggesting autoimmunological

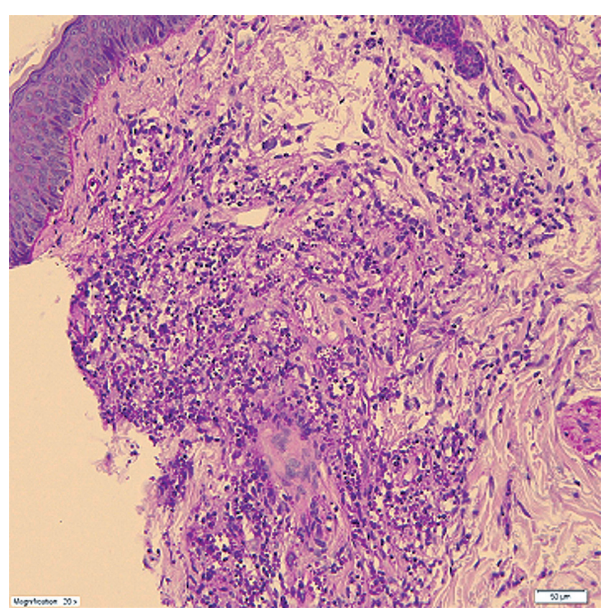

Figure 2. Staining with Alcian blue and mucicarmine affirms presence of cryptococcal organisms 
background), pancreas and in the lungs. Because of the suspicion of fungal infection in one of the patient's kidney's cysts, she underwent nephrectomy in June 2015 The patient required re-laparotomy due to bleeding as she suffered from multiorgan failure and coagulopathy. Despite aggressive multi-targeted treatment and many surgical procedures, the patient died 4 months after admission to the hospital. In the autopsy examination cryptococcal colonies were found not only in the kidneys, but also in the thyroid and pancreas, with no sign of them in the lungs. According to the post-mortem examination, the circulatory failure, being a result of a fungal infection of the kidney, thyroid and pancreas, led to a septic shock, and was a direct cause of death.

Further research is needed to assess all the possible organs that may be involved in cryptococcal infections.

\section{Diagnosis}

Histopathological examination assessed from skin biopsy specimens with microscopic evaluation and tissue culture are crucial steps for the diagnosis of cryptococcosis. C. neoformans can be grown on various types of microbiological media with optimal temperature of growth between 30 and $3^{\circ} \mathrm{C}$ and $40^{\circ} \mathrm{C}$ as maximum tolerated one [18]. Presence of mucoid colonies is a typica feature of this fungus and is usually the first clue that brings the suspicion of cryptococcal infection. Furthermore, $C$. neoformans can be observed as round, encapsulated yeast-like budding cells with 5 to $10 \mu \mathrm{m}$ in diameter [19]. After staining with classic haematoxylin and eosin stains, clear space around cells can be seen, suggesting appearance of the capsule. PAS stains decorate the yeast and mucicarmine stain decorates the highly characteristic gelatinous capsule a bright pink. Moreover, Grocott methenamine silver, toluidine blue or methylene blue stain are sometimes helpful to depict characteristic budding yeasts. To prove their presence, staining with Alcian blue and Mayer's or Southgate's mucicarmine can be performed. There are also many biochemical tests in commercially available kits for clinical laboratories. Cryptococcal polysaccharide antigen can be detected in CSF and serum via latex agglutination test or by ELISA [20].

It is crucial to confirm or rule out disseminated cryptococcosis so blood, urine and CSF should be examined, however antigen titres in CSF and serum may be lower in HIV-negative patients than in HIV-positive ones with CNS cryptococcosis. Disseminated cryptococcosis needs a positive culture from at least two different sites or a positive blood culture to be confirmed [21, 22].

\section{Treatment}

Due to the risk of developing meningoencephalitis, the drugs used in treatment of cryptococcosis ought to be the ones that penetrate the blood-brain barrier. As stated in the guidelines for the management of cryptococcosis by the Infectious Diseases Society of America (IDSA) in 2010, treatment choice depends on the patient's immune status. Another important factor is the location of the infection. It is especially important to rule out CNS disease in disseminated cryptococcosis. IDSA recommends induction therapy for 2 weeks, which consists of liposomal amphotericin B in a daily dose 3-4 mg/kg intravenously or amphotericin B lipid complex $5 \mathrm{mg} / \mathrm{kg} /$ day in co-therapy with flucytosine $100 \mathrm{mg} /$ day [23]. As consolidation therapy fluconazole should be administered in 400-800 mg dose per day for 8 weeks. Subsequently, fluconazole's dose should be reduced to 200-400 mg/day and continued from 6 months to 1 year.

Due to amphotericin's risk of nephrotoxicity both renal function and dosages should be carefully monitored. Serum concentration of flucytosine should be also monitored because of its gastrointestinal toxicity and bone marrow suppression. It is also crucial in SOT recipients to carefully plan a gradual reduction of the immunosuppressive treatment during antifungal therapy so that the graft functions properly and the infection is being eradicated at the same time.

Unfortunately, despite common antifungal treatment and many other medical measures, mortality in patients with cryptococcosis remains high. In Cryptococcus neoformans, resistance to azoles may be associated with alterations in the target enzyme encoded by the gene ERG11, lanosterol $14 \alpha$-demethylase [24]. These alterations are obtained through mutations or by overexpressing the gene encoding.

Recent evidence suggests that the majority of infections produced by this pathogen are associated with biofilm growth, which is also related with increased resistance to antifungal agents. Therefore, there is a great need to search for alternative antifungal agents for these fungi. The search for new molecules is currently occurring from nanoparticle drugs of plant peptide origin [25].

\section{Discussion}

Invasive fungal infections are still a huge threat and they concern around $15-42 \%$ of organ transplant recipients [26]. The overall occurrence of C. neoformans infection in SOT recipients reaches $2.8 \%$ and makes it the third most common invasive fungal infection in patients after transplantations with mortality of $42 \%$ [6]. Cryptococcosis develops in a median of 21 months after transplantation and according to another study, the median time to onset after kidney transplantation is 35 months [11]. Fifty-nine percent of cases occurred more than 12 months after transplantation. In case of our patient, it was only 3 months from the renal transplantation to disseminated cryptococcosis, which was most probably a result of leucopenia and higher than usual doses of steroids administered to maintain a sufficient immunosuppression level after MMF reduc- 
tion. It has been proven that the type of adjusted immunosuppressive regimen may influence the outcome and clinical presentation of cryptococcosis in transplant recipients [10-12]. Patients who received calcineurin inhibitor-based treatment (tacrolimus or cyclosporine) as a first-line immunosuppressive regimen after transplantation were less likely to have disseminated disease including CNS involvement than those receiving non-calcineurin inhibitor-based regimen [6]. Cyclosporine $A$ (CSA) and tacrolimus (Tac) inhibit signal transduction which is necessary for T-cell production. Moreover, they present antifungal activity against C. neoformans as they inhibit fungal analogues of calcineurin. They are toxic to $\mathrm{C}$. neoformans at $37^{\circ} \mathrm{C}$ whereas in $24^{\circ} \mathrm{C}$ not [19].

Cutaneous cryptococcosis concerns around 10-20\% of patients with disseminated cryptococcosis and due to haematogenous spread and almost any type of skin lesion can be observed [20].

It is crucial to confirm or rule out disseminated cryptococcosis so blood, urine and CSF should be examined, however antigen titres in CSF and serum may be lower in HIV-negative patients than in HIV-positive ones with CNS cryptococcosis. Meningoencephalitis can be established by positive culture from the CSF and staining with India ink. In our patient, histopathological examination of the removed graft also demonstrated many fungal colonies after Grocott staining corresponding to cryptococcosis diagnosis.

The overall treatment of our patient was several times more intensive than guidelines indicate, lipid formulation of amphotericin B was administered for 64 days and flucytosine for 58 days, doses adjusted to both renal and liver function and haematological abnormalities (thrombocytopenia). Fluconazole was applied for 37 days, which is a relatively short time, but its discontinuation was due to lack of effectiveness and progressive worsening of the patient's condition. Pre-existent renal failure is a significant prognostic factor in transplant recipients infected with Cryptococcus $[1,27]$. Additionally advanced age, malnutrition and progressive graft function deterioration are the factors hindering the treatment.

\section{Conclusions}

Summarising, our case and the literature show that cryptococcosis persists to be serious complication of organ transplantations. Atypical skin lesions observed in immunosuppressed patients can be the first symptoms of $C$. neoformans infection, therefore cryptococcosis should always be a part of differential diagnosis in such cases and raise physicians' awareness.

The existence of primary cutaneous cryptococcosis is doubted. Hence skin manifestations should always imply systemic involvement and appropriate systemic treatment should be implemented.
In case of the patient from our Clinic, cryptococcosis evolved only within 3 months after the transplantation. An incident of leucopenia with no antifungal prophylaxis, prolonged antibiotic treatment and intensive steroid treatment due to acute rejection suspicion led to higher susceptibility towards cryptococcal infection.

PET examination result showed an unusual active metabolic process in the thyroid and pancreas, where C. neoformans was found in the post mortem examination, so we suggest that PET should be a part of diagnosis in difficult cases. Even though fluconazole is not routinely recommended to patients after kidney transplantation, it should be introduced in patients with leucopenia. It is also worth highlighting our patient's dermatological manifestations of the cutaneous cryptococcosis - solid papules, hardly resembling anything, cutaneous involvement which we have not found in any of the cases available in the literature.

\section{Conflict of interest}

The authors declare no conflict of interest.

\section{References}

1. Chayakulkeeree M, Perfect JR. Cryptococcosis. Infect Dis Clin North Am 2006; 20: 507-44.

2. Perfect JR, Casadevall A. Cryptococcosis. Infect Dis Clin North Am 2002; 16: 837-74.

3. Stąpór W, Rancewicz Z, Zegadło-Mylik M, et al. Cutaneous cryptococcosis after renal transplantation - report of two cases. Przegl Dermatol 2000; 87: 153-7.

4. Neuville S, Dromer F, Morin O, et al. Primary cutaneous cryptococcosis: a distinct clinical entity. Clin Infect Dis 2003; 36 : 337-47.

5. Singh N, Alexander BD, Lortholary O, et al. Cryptococcus neoformans in organ transplant recipients: impact of calcineurininhibitor agents on mortality. J Infect Dis 2007; 195: 756-64.

6. Husain S, Wagener MM, Singh N. Cryptococcus neoformans infection in organ transplant recipients: variables influencing clinical characteristics and outcome. Emerg Infect Dis 2001; 7: 375-81.

7. Negroni R. Cryptococcosis. Clin Dermatol 2012; 30: 599-609.

8. Diamond RD, May JE, Kane MA, et al. The role of the classical and alternative complement pathways in host defense against Cryptococcus neoformans infection. J Immunol 1974; 112: 2260-70.

9. Badreshia S, Klepeiss S, Ioffreda M, et al. Cutaneous cryptococcus in an elderly woman with chronic essential dermatitis. Cutis 2006; 78: 53-6.

10. Schroter GP, Temple DR, Husberg BS, et al. Cryptococcosis after renal transplantation: report of ten cases. Surgery 1976; 79: 268-77.

11. Jarvis JN, Harrison TS. Pulmonary cryptococcosis. Semin Respir Crit Care Med 2008; 29: 141-50.

12. Subramanian S, Mathai D. Clinical manifestations and management of cryptococcal infection. J Postgrad Med 2005; 51 Suppl S1: 21-6.

13. Al-Akeel R, Ahmed M, Syed R. An overview of diagnostic criteria for identification of cryptococcal meningitis with special emphasis on AIDS. African J Biotechnol 2012; 11: 11760-6. 
14. Martinez LR, Garcia-Rivera J, Casadevall A. Cryptococcus neoformans var. neoformans (serotype D) strains are more susceptible to heat than C. neoformans var. grubii (serotype A) strains. J Clin Microbiol 2001; 39: 3365-7.

15. Latino GA, Gago E, Vidau P, Vivanco B. Cutaneous cryptococcosis in a patient on chronic haemodialysis. Nefrologia 2012; 32: 697-8.

16. Schupbach CW, Wheeler CE, Briggaman RA, et al. Cutaneous manifestations of disseminated cryptococcosis. Arch Dermatol 1976; 112: 1734-40.

17. Badreshia S, Klepeiss S, loffreda M, et al. Cutaneous cryptococcus in an elderly woman with chronic essential dermatitis. Cutis 2006; 78: 53-6.

18. Gazzoni AF, Severo CB, Salles EF, Severo LC. Histopathology, serology and cultures in the diagnosis of cryptococcosis. Rev Inst Med Trop Sao Paulo 2009; 51: 255-9.

19. Odom A, Muir S, Lim E, et al. Calcineurin is required for virulence of Cryptococcus neoformans. EMBO J 1997; 16: 2576-89.

20. Valente ES, Lazzarin MC, Koech BL, et al. Disseminated cryptococcosis presenting as cutaneous cellulitis in an adolescent with systemic lupus erythematosus. Infect Dis Rep 2015; 7: 5743.

21. Chuang YM, Ho YC, Chang HT, et al. Disseminated cryptococcosis in HIV-uninfected patients. Eur J Clin Microbiol Infect Dis 2008; 27: 307-10.

22. Mostafa WZ, Ishak EA, Ekladious EM, Arnaout HH. Disseminated cryptococcosis with cutaneous lesions. J Dermatol 1996; 23: 209-13.

23. Perfect JR, Dismukes WE, Dromer F, et al. Clinical practice guidelines for the management of cryptococcal disease: 2010 update by the Infectious Diseases Society of America. Clin Infect Dis 2010; 50: 291-322.

24. Ricchi E, Manfredi R, Scarani P, et al. Cutaneous cryptococcosis and AIDS. J Am Acad Dermatol 1991; 25: 335-6.

25. Gullo FP, Rossi SA, Sardi JD, et al. Cryptococcosis: Epidemiology, fungal resistance, and new alternatives for treatment. Eur J Clin Microbiol Infect Dis 2013; 32: 1377-91.

26. Singh N, Alexander BD, Lortholary O, et al. Cryptococcus neoformans in organ transplant recipients: impact of calcineurininhibitor agents on mortality. J Infect Dis 2007; 195: 756-64.

27. Christianson JC, Engber W, Andes D. Primary cutaneous cryptococcosis in immunocompetent and immunocompromised hosts. Med Mycol 2003; 41: 177-88. 